\title{
VALUTAZIONE DELLA QUALITÀ DI DISTRIBUZIONE DI DIFFERENTI MISCELE DI FERTILIZZANTI SOLIDI
}

\author{
Paolo Balsari, Elio Dinuccio, Mario Tamagnone
}

\section{INTRODUZIONE}

\section{Introduction}

Tra gli aspetti inerenti la gestione delle colture agrarie, quelli relativi ad una corretta fertilizzazione minerale sono di primaria importanza. Applicazioni di concimi in dosi insufficienti o eccessivamente elevate, in parte o su tutta la superficie, possono tradursi non solo in differenti risposte produttive ma anche in fenomeni di tossicità e in una maggiore predisposizione delle colture alle malattie. Parallelamente, l'impiego di concimi chimici in quantità eccedente rispetto alle reali necessità può dare luogo a problemi di carattere ambientale [11]. Quest'ultimo aspetto, alla luce di un quadro normativo molto articolato e di una sempre maggiore attenzione verso le tematiche di carattere ambientale, riveste oggi un interesse crescente. Per far fronte a queste esigenze, negli ultimi anni l'attenzione dei ricercatori è stata rivolta allo studio di soluzioni operative in grado di limitare l'impatto ambientale della fertilizzazione minerale sia attraverso la messa a punto di dispositivi elettronici che, montati sullo spandiconcime, sono in grado di migliorare le prestazioni di tali macchine [9], sia attraverso la realizzazione e commercializzazione di nuove formulazioni complesse (miscele fisiche e chimiche) contenenti azoto a lento rilascio.

Queste ultime, in particolare, essendo caratterizzate da un titolo composto di elementi fertilizzanti consentono, da un lato di ridurre il numero degli interventi di distribuzione ed i costi a loro connessi, dall'altro di conseguire vantaggi ambientali legati ad una maggiore efficienza di utilizzazione dell'azoto distribuito [13]. Tuttavia mentre le miscele chimiche di differenti elementi fertilizzanti (bulk blend) garantiscono sino

Memoria presentata il 17.01.2006; accettata il 15.06.2006

Prof. Paolo Balsari, Prof. Ordinario di Meccanica Agraria (paolo.balsari@unito.it); Dr. Elio DinUCCIO, Dottorando di Ricerca; Dr. Mario Tamagnone, Ricercatore Confermato. Dipartimento di Economia e Ingegneria Agraria, Forestale e Ambientale - sezione di Meccanica, Università di Torino. allo spandimento la medesima composizione fisicochimica della massa, le miscele fisiche (blended) possono dare origine, a causa delle differenze di densità e diametro dei granuli che li compongono, ad una non ottimale uniformità di distribuzione $[5,12]$ dovuta a:

- fenomeni di stratificazione del prodotto nella tramoggia;

- diverse proprietà balistiche dei granuli.

In particolare, mentre il primo inconveniente si traduce in una limitata uniformità di distribuzione degli elementi fertilizzanti contenuti nella miscela, in senso sia trasversale che longitudinale all'avanzamento dello spandiconcime, il secondo ha un'influenza diretta solo sull'uniformità di distribuzione trasversale all'avanzamento dello spandiconcime [3].

L'entità di entrambi tali aspetti è strettamente legata alle caratteristiche fisiche della miscela, alle caratteristiche costruttive e al principio di funzionamento dello spandiconcime [10]. Da qui l'esigenza di poter disporre da un lato di macchine specificatamente realizzate e dall'altra di formulazioni fertilizzanti con caratteristiche fisiche ben definite. In tale contesto, il presente studio esamina quattro differenti tipi di concimi blended disponibili sul mercato con l'obbiettivo di fornire un contributo alla definizione dei principali parametri fisici che determinano la separazione dei granuli nella tramoggia e, contestualmente, mettere in rilievo gli eventuali limiti d'impiego dei concimi blended nella pratica agricola. È quest'ultimo principio che ha guidato la scelta di impiegare per le prove uno spandiconcime a distribuzione centrifuga che, come è noto, rappresenta il tipo di macchina maggiormente utilizzata in Europa.

\section{MATERIALI E METODI}

\section{Materials and methods}

Di ciascuno dei quattro concimi blended (Tabella 1) sono state determinate:

A. caratteristiche fisiche

B. qualità della distribuzione. 


\begin{tabular}{|c|c|c|}
\hline Concime & Titolo N-P-K & $\mathrm{N}^{\circ}$ frazioni \\
\hline A & $25-5-10$ & 4 \\
\hline B & $20-5-10$ & 4 \\
\hline C & $13-5-22$ & 3 \\
\hline D & $15-0-29$ & 2 \\
\hline
\end{tabular}

TABELLA 1 - Miscele fisiche oggetto delle prove.

TABLE 1 - Blended fertilizers tested.

\subsection{CARATTERISTICHE FISICHE}

\subsection{Physical properties}

Per ciascun concime sono stati determinati:

- la granulometria (dimensione dei granuli e classi di distribuzione) della miscela e di ciascuna frazione;

- la percentuale ponderale delle diverse frazioni;

- la massa volumica.

Per determinare le caratteristiche fisiche sono stati esaminati tre campioni per ogni tipo di concime considerato. Ogni campione, del peso di $1000 \mathrm{~g}$, è stato prelevato a caso da differenti confezioni commerciali (sacco da $20 \mathrm{~kg}$ ). Nel corso di queste determinazioni è stata utilizzata una bilancia elettronica con sensibilità $0,01 \mathrm{~g}$.

\subsubsection{GRANULOMETRIA - Granular distribution}

I campioni di fertilizzante tal quali e quelli delle singole frazioni sono stati suddivisi, per scuotimento in un setaccino in plexiglass a maglie variabili, nelle seguenti classi granulometriche:

- $<2,0 \mathrm{~mm}$

- da 2,0 a $3,3 \mathrm{~mm}$

- da 3,4 a $4,7 \mathrm{~mm}$

- $>4,7 \mathrm{~mm}$

2.1.2 Percentuale PONDERALE DELle FRAZIONI Weight percentage of the components

Ogni campione è stato suddiviso manualmente in singole frazioni appartenenti alla medesima classe cromatica. Di ciascuna delle singole frazioni cosi ottenute è stato determinato il peso.

\subsubsection{MASSA VOLUMiCA - Bulk density}

La massa volumica dei fertilizzanti tal quali e delle loro singole frazioni è stata ottenuta secondo quanto previsto dalla EN1236 [6]. In particolare, è stato impiegato un contenitore cilindrico con diametro di $100 \mathrm{~mm}$ e capacità di $1 \mathrm{dm}^{3}$. Il fertilizzante in esame è stato introdotto all'interno del cilindro fino al raggiungimento della sua capacità massima e, successivamente, si è determinato il peso del materiale presente nel cilindro. La massa volumica è stata determi-

nata come rapporto fra la massa introdotta e il volume del cilindro.

\subsection{QuaLitÀ DELLA DISTRIBUZIONE}

\subsection{Distribution quality}

La qualità della distribuzione è stata valutata esaminando i seguenti parametri:

- portata del concime in uscita dalla tramoggia;

- uniformità di distribuzione sia longitudinale che trasversale all'avanzamento dello spandiconcime;

- separazione dei granuli durante la distribuzione.

Le prove sono state condotte utilizzando uno spandiconcime bidisco KUHN MDS 61 a distribuzione centrifuga, con tramoggia troncoconica e capacità di $350 \mathrm{l}$, portato da una trattrice della potenza di $60 \mathrm{~kW}$. Il sistema di distribuzione dello spandiconcime è costituito da due dischi controrotanti, azionati per mezzo della presa di potenza, ciascuno con diametro di $320 \mathrm{~mm}$ e dotati di alette radiali; la distanza fra gli assi di rotazione dei dischi è pari a $440 \mathrm{~mm}$. Il flusso del concime, dalla tramoggia verso il sistema di distribuzione, avviene per gravità ed è regolato manualmente attraverso l'apertura di due luci disposte sul fondo della tramoggia in prossimità dei dischi di distribuzione.

Sul fondo della tramoggia è presente un agitatore meccanico in grado di evitare l'impaccamento del concime in prossimità delle luci di uscita.

La sperimentazione è stata effettuata su un terreno pianeggiante situato nel comprensorio della Facoltà di Agraria di Grugliasco (TO).

La trattrice è stata impiegata con regime di rotazione della presa di potenza di 450 giri $\min ^{-1}$ e velocità di avanzamento di $4,0 \mathrm{~km} \mathrm{~h}^{-1}$; lo spandiconcime con una velocità di rotazione dei dischi di 788 giri $\mathrm{min}^{-1}$, un'altezza dei dischi dal suolo di $650 \mathrm{~mm}$ e con i dischi in posizione orizzontale.

Per ogni concime sono state impiegate due dosi di distribuzione ( 250 e $500 \mathrm{~kg} \mathrm{ha}^{-1}$ ) e 3 livelli di riempi-

\begin{tabular}{|c|c|c|}
\hline Tesi & $\begin{array}{c}\text { Dose di } \\
\text { distribuzione } \\
\left(\mathrm{kg} \mathrm{ha}^{-1}\right)\end{array}$ & $\begin{array}{c}\text { Livello riempimento } \\
\text { della tramoggia } \\
(\%)\end{array}$ \\
\hline 1 & 250 & 90 \\
\hline 2 & 250 & 50 \\
\hline 3 & 250 & 10 \\
\hline 4 & 500 & 90 \\
\hline 5 & 500 & 50 \\
\hline 6 & 500 & 10 \\
\hline
\end{tabular}

TABELLA 2 - Schema sperimentale adottato per ogni concime in prova.

TABLE 2 - Experimental scheme used for each type of tested fertilizers. 
mento della tramoggia (Tabella 2) effettuando tre ripetizioni. I tre differenti livelli di riempimento sono stati ottenuti effettuando la distribuzione in normali condizioni operative per evidenziare eventuali fenomeni si separazione delle diverse frazioni componenti la miscela fisica.

I dati ottenuti sono stati elaborati statisticamente mediante ANOVA UNIVARIATA $(\mathrm{p}=0,05)$.

\subsubsection{PortatA IN USCITA DALLA TRAMOGGIA - Out-} going flow rate

La portata del concime in uscita dalla tramoggia varia, per lo spandiconcime impiegato, in funzione della luce di uscita dalla tramoggia e delle caratteristiche fisiche del concime. Essa è stata determinata in condizioni statiche, togliendo il disco di distribuzione e raccogliendo il concime caduto dalla tramoggia per un tempo di $60 \mathrm{~s}$.

La determinazione della correlazione tra portata e apertura della luce di uscita dalla tramoggia ha consentito di individuare, per ciascuno dei quattro concimi oggetto delle prove, la posizione di apertura in grado di fornire la dose di distribuzione desiderata (250 $\left.500 \mathrm{~kg} \mathrm{ha}^{-1}\right)$.

\subsubsection{UNIFORMITÀ DI DISTRIBUZIONE - Distribution} evenness

L'uniformità di distribuzione trasversale all'avanzamento dello spandiconcime è stata determinata secondo quanto indicato dalla EN 13739-2 [8]. Sono state utilizzate delle cassette di materiale plastico, dotate internamente di 8 setti di separazione, delle dimensioni di $0,50 \times 0,50 \times 0,15 \mathrm{~m}$ (Fig. 1).

Le cassette sono state poste sul terreno, lungo una linea trasversale a quella di avanzamento dello spandiconcime, ad una distanza reciproca di 1,0 m (Fig. 2). La cassetta centrale, per consentire il passaggio della trattrice, è stata posizionata ad una distanza di $1,5 \mathrm{~m}$ da quelle adiacenti laterali.

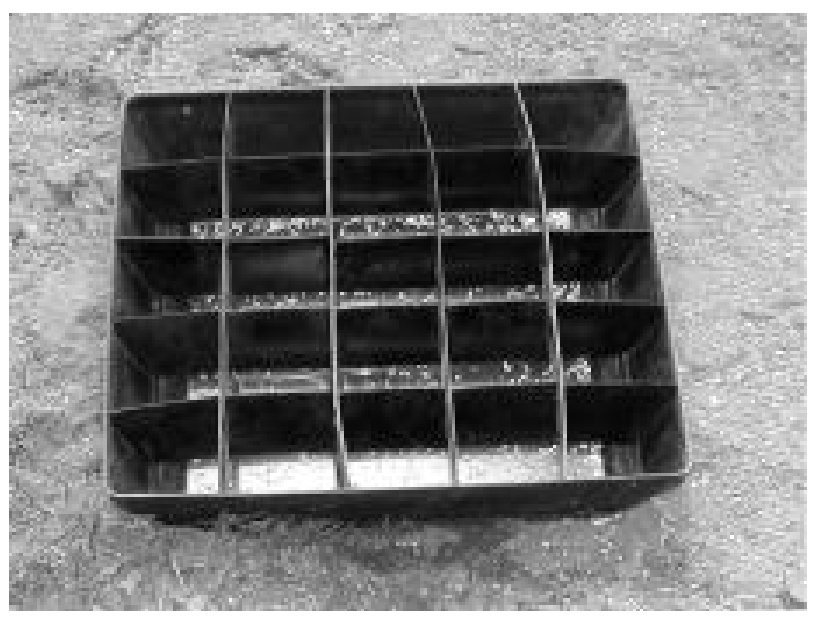

Fig. 1 - Cassetta utilizzata per le prove di uniformità trasversale.

Fig. 1 - Box used for the tests of transversal evenness distribution.

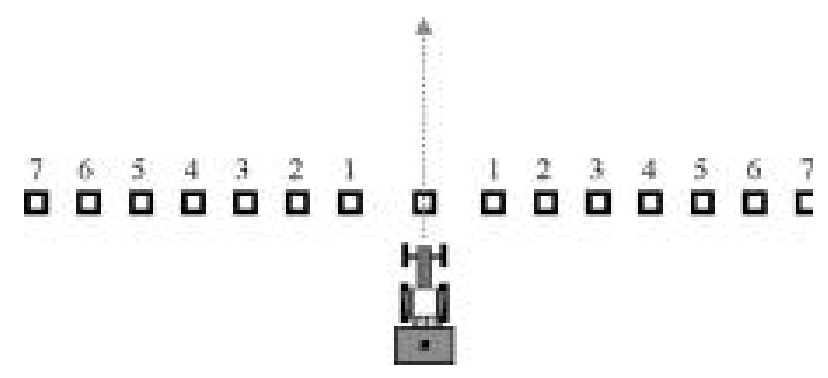

Fig. 2 - Schema di posizionamento delle cassette adottato nelle prove.

Fig. 2 - Scheme of the boxes disposal used in the trial.

Il materiale caduto all'interno di ogni cassetta è stato raccolto, separato nelle diverse frazioni cromatiche che sono state, successivamente, pesate per mezzo di una bilancia elettronica (sensibilità $0,01 \mathrm{~g}$ ). I valori ottenuti sono stati elaborati attraverso un software messo a punto dalla sezione di Meccanica del DEIAFA [2] che ha permesso di ottenere:

- i diagrammi di distribuzione del concime tal quale;

- i diagrammi di distribuzione per singolo componente;

- i coefficienti di variazione (CV) della risultante della distribuzione;

- la larghezza di lavoro ottimale.

In particolare il software, partendo dal diagramma di distribuzione trasversale dello spandiconcime, determina l'uniformità di distribuzione raggiungibile con diverse larghezze di lavoro. La larghezza di lavoro ottimale, quella cioè che consente il più ampio fronte di lavoro pur garantendo una sufficiente uniformità di distribuzione, è stata determinata mediante l'analisi del CV della risultante ottenuta con più passaggi contigui (nel calcolo vengono considerate solo le zone con una corretta sovrapposizione). Nella presente ricerca sono stati calcolati i valori di CV relativi alle larghezze di lavoro comprese tra 4 e $10 \mathrm{~m}$ ed è stato considerato come valore limite accettabile un CV pari al 15\% come previsto dalla EN 13793-1 [7].

Per evidenziare eventuali differenze del diagramma di distribuzione durante lo svuotamento della tramoggia nel corso della distribuzione, è stato calcolato un valore indice, indice di differenza tra i diagrammi (ID), che esprime le differenze fra i profili dei diagrammi di distribuzione ottenuti ai tre livelli di riempimento della tramoggia $(90 \%, 50 \%$ e $10 \%)$. Questo indice è dato dalla somma delle differenze tra i valori di massimo e minimo rilevati per i tre livelli di riempimento della tramoggia in ogni punto del diagramma di distribuzione:

$$
\mathrm{ID}=\sum_{n=1}^{15}(\text { valore } \max -\text { valore } \min )
$$

In particolare, ridotti valori dell'indice indicano che i diagrammi sono molto simili tra loro mentre, al contrario, valori elevati denotano differenze fra i diagrammi durante lo svuotamento della tramoggia. 
2.2.3 SePARAZIONE DEI GRANULI DURANTE LA DISTRIBUZIONE - Granules segregation during spreading

La procedura utilizzata, per indagare l'eventuale separazione dei granuli durante la distribuzione dei concimi blended, è consistita in:

- separazione nelle diverse frazioni, precedentemente definite, del concime contenuto in ogni cassetta;

- pesatura delle singole frazioni mediante una bilancia elettronica con sensibilità $0,01 \mathrm{~g}$;

- elaborazione dei valori ottenuti utilizzando il programma di calcolo del DEIAFA [2];

- determinazione di un coefficiente di separazione (CS) calcolato attraverso la somma delle differenze, in valore assoluto, tra i CV (calcolati sulla risultante della sovrapposizione di più passaggi contigui) delle diverse frazioni di concime e il CV del concime tal quale ottenuto alla larghezza di lavoro ottimale:

$\mathrm{CS}=\sum_{i=1}^{n} \mathrm{ICV}$ frazione $_{\mathrm{i}}-\mathrm{CV}$ tal qualel

Per ogni concime, mediando opportunamente i valori indice e il coefficiente di separazione ottenuti, è stato possibile fare delle considerazioni sulla qualità della distribuzione riguardo:

- il tipo di concime impiegato (facendo la media di tutti i valori indice ottenuti con le diverse variabili esaminate);

- il livello di riempimento della tramoggia;

- la dose distribuita (facendo la media dei coefficienti di separazione ottenuti, per ogni concime, ai tre livelli di riempimento e per ognuna delle due dosi di distribuzione);

- l'interazione dose / tipo di concime;

- l'interazione livello di riempimento / tipo di concime.

\section{RISULTATI E DISCUSSIONE}

\section{Results and discussion}

\subsection{CARATTERISTICHE FISICHE}

\subsection{Physical properties}

\subsubsection{GRANULOMETRIA - Granular distribution}

In nessuno dei fertilizzanti impiegati nelle prove sono stati rilevati granuli di diametro superiore a $4,7 \mathrm{~mm}$.

\begin{tabular}{|c|c|c|c|c|}
\hline \multirow{2}{*}{ Concime } & \multicolumn{4}{|c|}{ Dimensioni (mm) } \\
\cline { 2 - 5 } & $<2,0$ & $2,0-3,3$ & $3,4-4,7$ & $>4,7$ \\
\hline A & 10,3 & 84,0 & 5,7 & 0,0 \\
\hline B & 10,2 & 88,3 & 1,5 & 0,0 \\
\hline C & 5,7 & 91,7 & 2,6 & 0,0 \\
\hline D & 100,0 & 0,0 & 0,0 & 0,0 \\
\hline
\end{tabular}

TABELLA 3 - Ripartizione percentuale per classi granulometriche dei concimi oggetto delle prove.

TABLE 3 - Granular size distribution of the tested fertilizers.
Le dimensioni dei granuli dei fertilizzanti A, B e C sono risultate prevalentemente comprese tra $2,0 \mathrm{e}$ $3,3 \mathrm{~mm}$, e solo in limitata parte nelle classi $<2,0 \mathrm{~mm}$ e fra 3,4 e $4,7 \mathrm{~mm}$ (Tabella 3). Diversamente, il concime $\mathrm{D}$ è risultato costituito per il $100 \%$ da granuli di dimensioni inferiori a $2,0 \mathrm{~mm}$.

\subsubsection{Percentuale Ponderale delle FRAZioni -} Weight percentage of the components

Il rapporto fra le diverse frazioni dei singoli concimi è risultato molto variabile. Per quanto riguarda il concime A si ha una frazione preponderante $(56 \%)$ accompagnata da altre 3 ripartite in maniera abbastanza omogenea. Il concime $\mathrm{B}$ è costituito da una frazione che rappresenta il $37 \%$ della massa, una l' $11 \%$ e due circa il $25 \%$. Nel concime $\mathrm{C}$ le 3 frazioni sono rappresentate in misura poco diversa, mentre il concime $\mathrm{D}$ è costituito da 2 frazioni di cui una rappresenta i $3 / 4$ della massa totale (Tabella 4).

Non sono state rilevate particolari differenze fra la granulometria della miscela e quella delle singole frazioni, eccetto che per la frazione bianca del concime $\mathrm{C}$ che risulta costituita da granuli di maggiori dimensioni.

\subsubsection{Massa Volumica - Bulk density}

La massa volumica delle miscele esaminate è risultata compresa tra 0,89 e $1,05 \mathrm{~kg} \mathrm{dm}^{-3}$, rispettivamente per i concimi A e C.

Esaminando i valori relativi alle singole frazioni

\begin{tabular}{|c|c|c|c|c|c|}
\hline \multirow{4}{*}{ Concime } & \multirow{2}{*}{ Frazione } & \multirow{2}{*}{$\%$} & \multicolumn{3}{|c|}{ Dimensioni (mm) } \\
\cline { 4 - 6 } & & & $<2,0$ & $2,0-3,3$ & $3,4-4,7$ \\
\hline \multirow{4}{*}{ A } & giallo & 55,7 & 2,2 & 94,0 & 3,8 \\
\cline { 2 - 6 } & grigio & 15,8 & 7,8 & 85,5 & 6,7 \\
\cline { 2 - 6 } & marrone & 15,3 & 14,0 & 79,3 & 6,6 \\
\cline { 2 - 6 } & bianco & 13,2 & 6,9 & 86,7 & 6,3 \\
\hline \multirow{4}{*}{ B } & azzurro & 26,1 & 4,1 & 95,0 & 0,9 \\
\cline { 2 - 6 } & grigio & 37,1 & 4,1 & 89,4 & 6,6 \\
\cline { 2 - 6 } & marrone & 11,4 & 14,7 & 81,3 & 4,0 \\
\cline { 2 - 6 } & bianco & 25,4 & 7,7 & 84,6 & 7,7 \\
\hline \multirow{4}{*}{ C } & azzurro & 28,3 & 3,2 & 95,7 & 1,1 \\
\cline { 2 - 6 } & grigio & 44,4 & 8,5 & 85,2 & 6,3 \\
\cline { 2 - 6 } & bianco & 27,3 & 2,1 & 85,7 & 12,2 \\
\hline \multirow{4}{*}{ D } & arancione & 26,8 & 100,0 & 0,0 & 0,0 \\
\cline { 2 - 6 } & marrone & 73,2 & 100,0 & 0,0 & 0,0 \\
\hline \multirow{4}{*}{} & & & & & \\
\hline
\end{tabular}

TABella 4 - Componenti le miscele analizzate e loro classi granulometriche.

TABLE 4 - Components of the tested fertilizers and their granular size. 


\begin{tabular}{|c|c|}
\hline $\begin{array}{c}\text { Concime / } \\
\text { Frazione }\end{array}$ & $\begin{array}{c}\text { Massa } \\
\text { volumica } \\
\left(\mathrm{kg} \mathrm{dm}^{-3}\right)\end{array}$ \\
\hline $\mathrm{A}$ & 0,89 \\
\hline giallo & 0,68 \\
\hline grigio & 1,14 \\
\hline marrone & 0,96 \\
\hline bianco & 0,65 \\
\hline C & 1,05 \\
\hline azzurro & 0,77 \\
\hline grigio & 1,13 \\
\hline bianco & 0,86 \\
\hline
\end{tabular}

\begin{tabular}{|c|c|}
\hline $\begin{array}{c}\text { Concime / } \\
\text { Frazione }\end{array}$ & $\begin{array}{c}\text { Massa } \\
\text { volumica } \\
\left(\mathrm{kg} \mathrm{dm}^{-3}\right)\end{array}$ \\
\hline $\mathrm{B}$ & 0,96 \\
\hline azzurro & 0,80 \\
\hline grigio & 1,04 \\
\hline marrone & 1,09 \\
\hline bianco & 0,79 \\
\hline $\mathrm{D}$ & 1,04 \\
\hline arancione & 0,77 \\
\hline marrone & 1,29 \\
\hline
\end{tabular}

TABELla 5 - Massa volumica complessiva e delle diverse frazioni dei concimi oggetto delle prove.

TABLE 5 - Bulk density of the tested fertilizers.

dello stesso concime si evidenziano differenze di massa volumica molto elevate. La differenza fra la frazione più pesante e quella più leggera è risultata prossima al $40 \%$ per i concimi B e C e pari a circa il $70 \%$ per i concimi A e D (Tabella 5).

\subsection{Qualità DELLA DISTRIBUZIONE}

\subsection{Distribution quality}

3.2.1 REGOLAZIONE DEL SISTEMA DI DISTRIBUZIONE Distribution system adjustment

Correlando la portata del concime in uscita con l'apertura della tramoggia, sono state ottenute le equazioni che hanno consentito di individuare, per ciascuno dei quattro concimi, la posizione del regolatore in grado di fornire la dose di distribuzione desiderata con una larghezza di lavoro di $8 \mathrm{~m}$ (Tabella 6 ). In particolare i concimi $\mathrm{C}$ e $\mathrm{D}$, diversamente dai concimi A e B, hanno richiesto differenti regolazioni a causa, so-

\begin{tabular}{|c|c|c|}
\hline \multirow{2}{*}{ Concime } & $\begin{array}{c}\text { Dose } \\
\left(\mathrm{kg} \mathrm{ha}^{-1}\right)\end{array}$ & $\begin{array}{c}\text { Posizione del sistema di } \\
\text { regolazione }\end{array}$ \\
\hline \multirow{2}{*}{$\mathrm{A}$} & 250 & 20 \\
\cline { 2 - 3 } & 500 & 25 \\
\hline \multirow{2}{*}{$\mathrm{B}$} & 250 & 20 \\
\cline { 2 - 3 } & 500 & 25 \\
\hline \multirow{2}{*}{$\mathrm{C}$} & 250 & 18 \\
\cline { 2 - 3 } & 500 & 23 \\
\hline \multirow{2}{*}{$\mathrm{D}$} & 250 & 17 \\
\cline { 2 - 3 } & 500 & 22 \\
\hline
\end{tabular}

TABElla 6 - Posizione del sistema di regolazione in relazione alla dose di distribuzione.

TABLE 6 - Calibration device setting. prattutto, delle differenze di massa volumica e granulometria delle frazioni che li compongono.

\subsubsection{UNIFORMITÀ DI DISTRIBUZIONE - Distribution} evenness

Non sono emerse differenze significative fra i diagrammi di distribuzione nelle tre repliche effettuate $(\mathrm{P}>0,05)$ e pertanto, di seguito vengono riportati i valori medi. I diagrammi di distribuzione ottenuti evidenziano una forma triangolare tipica degli spandiconcime a reazione centrifuga a due dischi $[1,4]$. La gittata massima è risultata compresa fra i $10,0 \mathrm{~m}$ del concime D e i $14,0 \mathrm{~m}$ del concime C.

La determinazione dei coefficienti di variazione della risultante di passaggi contigui ha fatto registrare, per tutte le tesi analizzate, un andamento molto simile: a partire dalla larghezza di lavoro minima considerata $(4,0 \mathrm{~m})$ il valore del $\mathrm{CV}$ aumenta fino a raggiungere un picco intorno a $6,0 \mathrm{~m}$, segue una diminuzione del valore del CV fino a raggiungere un picco inferiore (che corrisponde alla larghezza di lavoro ottimale) per poi nuovamente aumentare fino alla massima larghezza di lavoro considerata $(10,0 \mathrm{~m})$ (Fig. 3).

Considerando i risultati ottenuti con la tramoggia riempita al $90 \%$ si hanno larghezze di lavoro ottimali pari a 7,0 m per i concimi B e D e di $8,0 \mathrm{~m}$ per i concimi A e C, indipendentemente dalla dose di distribuita. La distribuzione effettuata con il concime A ha fatto registrare dei coefficienti di variazione prossimi al limite di accettabilità: $14,4 \%$ con dose di $250 \mathrm{~kg} \mathrm{ha}^{-1}$ e $14,7 \%$ con dose di $500 \mathrm{~kg} \mathrm{ha}^{-1}$. Gli altri 3 tipi di concime hanno fatto registrare valori del CV inferiori (Tabella 7).

Nel corso dello svuotamento della tramoggia è stata riscontrata una variazione del profilo di distribuzione trasversale la quale ha avuto un'influenza diretta sull'uniformità di distribuzione. In particolare, in corrispondenza del livello di riempimento della tramoggia pari al 50\%, è stato riscontrato un aumento del $\mathrm{CV}$ per i concimi A e B alla dose di $250 \mathrm{~kg} \mathrm{ha}^{-1}$ e per il

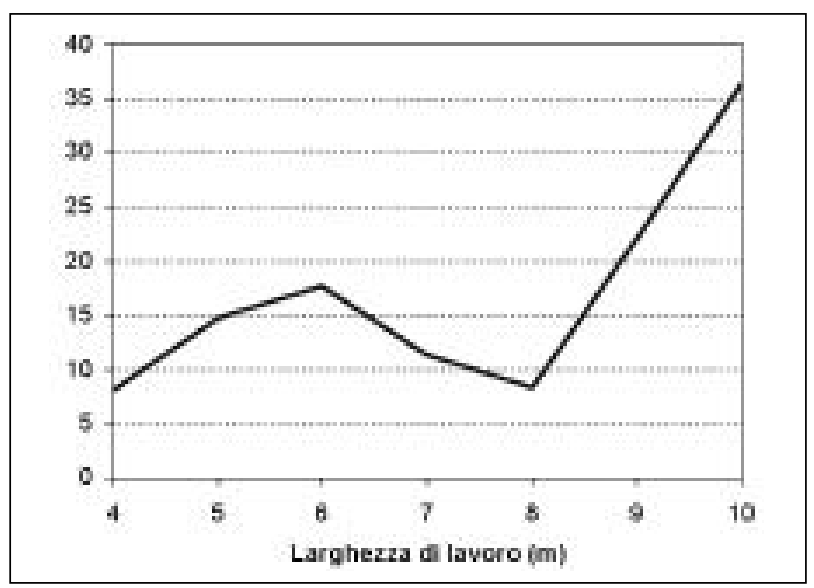

Fig. 3 - Uniformità di distribuzione in funzione della larghezza di lavoro.

Fig. 3 - Distribution evenness vs. working width. 


\begin{tabular}{|c|c|c|c|c|c|}
\hline \multirow{2}{*}{ Concime } & \multirow{2}{*}{$\begin{array}{c}\text { Larghezza } \\
\text { lavoro } \\
\text { (m) }\end{array}$} & \multirow{2}{*}{$\begin{array}{c}\text { Dose } \\
\left(\mathrm{kg} \mathrm{ha}^{-1}\right)\end{array}$} & \multicolumn{3}{|c|}{ Livello tramoggia } \\
\hline & & & $90 \%$ & $50 \%$ & $10 \%$ \\
\hline \multirow{2}{*}{ A } & \multirow{2}{*}{8,0} & 250 & 14,4 & 17,2 & 8,3 \\
\hline & & 500 & 14,7 & 12,9 & 11,2 \\
\hline \multirow{2}{*}{ B } & \multirow{2}{*}{7,0} & 250 & 12,3 & 17,5 & 15,1 \\
\hline & & 500 & 10,0 & 9,6 & 6,0 \\
\hline \multirow{2}{*}{$\mathrm{C}$} & \multirow{2}{*}{8,0} & 250 & 12,9 & 11,9 & 10,1 \\
\hline & & 500 & 13,2 & 7,5 & 6,5 \\
\hline \multirow{2}{*}{$\mathrm{D}$} & \multirow{2}{*}{7,0} & 250 & 13,7 & 14,0 & 14,2 \\
\hline & & 500 & 3,4 & 17,2 & 8,1 \\
\hline
\end{tabular}

TABELla 7 - Uniformità di distribuzione risultante $(C V)$ in funzione della dose e del livello di riempimento della tramoggia.

TABLE 7 - Distribution evenness vs. application rate and hopper level.

concime D con entrambi i dosaggi. In alcuni di questi casi il CV ha superato il limite di accettabilità (Tabella 7). Procedendo con lo svuotamento della tramoggia i valori del $\mathrm{CV}$ sono rientrati al di sotto del limite eccetto che per il concime B a $250 \mathrm{~kg} \mathrm{ha}^{-1}$.

Dall'analisi di questi dati si può evidenziare come sia la granulometria propria di ciascuno dei concimi oggetto delle prove, sia il livello di riempimento della tramoggia e la dose distribuita influiscono sulla forma del profilo di distribuzione e, quindi, sull'uniformità del diagramma risultante. In particolare, il diagramma ottenuto con il concime C (Fig. 4) risulta caratterizzato da un profilo più regolare e simmetrico e tale da fornire buone uniformità di distribuzione $(\mathrm{CV}<15 \%)$ in corrispondenza di larghezze di lavoro maggiori rispetto agli altri concimi e di consentire di mantenere una buona uniformità di distribuzione anche in prossimità di queste larghezze di lavoro. Ciò, nella pratica, consente all'operatore di commettere degli errori anche di una certa entità (1-2 m) nel mantenere la medesima distanza fra un passaggio dello spandiconcime e il successivo senza che ciò influisca in maniera consi-

\begin{tabular}{|c|c|c|}
\hline \multirow{2}{*}{ Concime } & \multicolumn{2}{|c|}{ Dose distribuita $\left(\mathrm{kg} \mathrm{ha}^{-1}\right)$} \\
\cline { 2 - 3 } & 250 & 500 \\
\hline A & 33,9 & 22,6 \\
\hline B & 22,1 & 12,1 \\
\hline C & 12,8 & 11,6 \\
\hline D & 9,5 & 17,3 \\
\hline
\end{tabular}

TABELla 8 - Valori dell'indice di differenza tra i diagrammi ottenuti.

TABLE 8 - Difference pattern index obtained.

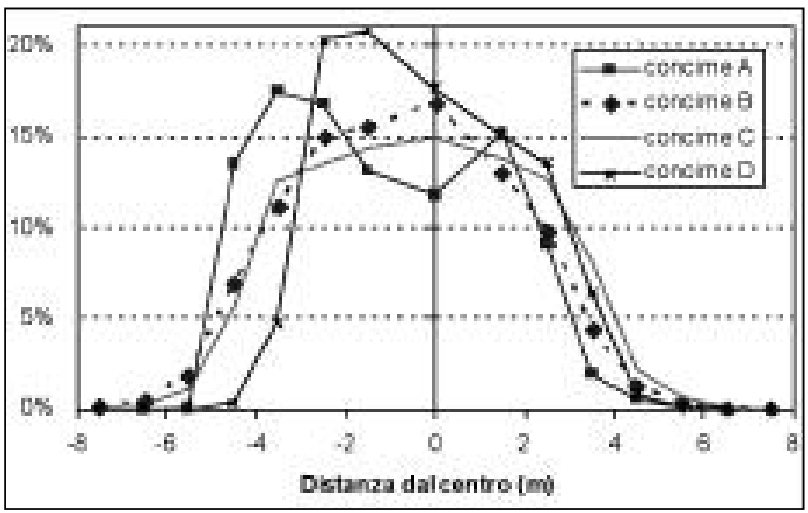

Fig. 4 - Diagrammi di distribuzione ottenuti con i diversi concimi esaminati.

Fig. 4 - Fertilizer pattern.

derevole sull'uniformità di distribuzione. Diversamente i concimi A e D caratterizzati da diagrammi di distribuzione più asimmetrici ed irregolari, a loro volta legati alle caratteristiche fisiche dei concimi (classi granulometriche più diversificate e maggiore presenza della classe granulometrica $<2 \mathrm{~mm}$ ), non sono risultati in grado, se non in corrispondenza di un limitato numero di larghezze di lavoro e per lo più ridotte, di fornire una buona uniformità di distribuzione impiegando lo spandiconcime a due dischi oggetto delle prove. In particolare, il concime A pur avendo un'elevata percentuale di granuli $(84 \%)$ nella classe 2,0 $3,3 \mathrm{~mm}$ non consente di creare un regolare e simmetrico diagramma di distribuzione a causa della presenza al suo interno della frazione di colore grigio caratterizzata da una massa volumica $\left(1,14 \mathrm{~kg} \mathrm{dm}^{-3}\right)$ ben differente rispetto a quella degli altri componenti la miscela (in media circa $0,75 \mathrm{~kg} \mathrm{dm}^{-3}$ ).

Per quel che riguarda l'indice di differenza tra i diagrammi, in generale, $i$ valori più elevati di quest'ultimo, che si ricorda, corrispondono ad una ridotta uguaglianza dei diagrammi, sono stati ottenuti operando a dose ridotta (Tabella 8) con eccezione del concime D che, con valore di indice di uguaglianza maggiore per la dose di distribuzione più alta, evidenzia, durante la distribuzione, un comportamento inverso rispetto agli altri tipi di concimi. Le differenze maggiori fra i diagrammi sono state ottenute con l'impiego del concime $\mathrm{A}$, mentre il concime $\mathrm{C}$ sembra essere quello che risente meno dell'effetto della dose di distribuzione.

\subsubsection{SEPARAZIONE DEI GRANULI DURANTE LA DISTRI-} BUZIONE - Granules segregation during spreading

I diagrammi di distribuzione delle singole frazioni di concime presentano andamenti simili a quelle del diagramma del concime tal quale. Il diverso comportamento delle frazioni rispetto al concime tal quale emerge analizzando i coefficienti di separazione. Il valore medio dei coefficienti è risultato pari a 9,5 con un campo di variazione compreso fra un minimo (ri- 
dotta separazione) di 0,7 (concime $D$ alla dose di distribuzione di $250 \mathrm{~kg} \mathrm{ha}^{-1}$ e livello di riempimento della tramoggia al 90\%) e un massimo di 35,0 (conci$m e \mathrm{~B}$ alla dose di distribuzione di $500 \mathrm{~kg} \mathrm{ha}^{-1}$ e livello di riempimento della tramoggia al $10 \%$ - Tabella 9).

I risultati ottenuti dalle medie dei coefficienti di separazione relativi alle variabili esaminate, hanno permesso di evidenziare che il concime che si separa di più nella tramoggia è quello denominato con la lettera B (media del coefficiente 17,1), segue il concime A (media del coefficiente 11,4), il $C$ (media del coefficiente 6,7 ) e il $D$ (media del coefficiente 2,6). I valori ridotti del coefficiente di separazione ottenuti impiegando il concime D sono attribuibili alla sua uniformità granulometrica e al limitato numero di frazioni di cui è composto.

Esaminando i dati relativi alla dose di distribuzione emerge che tale parametro ha una limitata interferenza sulla separazione delle frazioni.

Diversamente, la separazione aumenta man mano che la tramoggia si svuota: il coefficiente medio di separazione sale da circa 6 (livello 90\%) fino ad oltre 13 (livello 10\%). Ciò è legato al maggior tempo di permanenza del concime nella tramoggia che permette un maggior movimento reciproco dei granuli delle diverse frazioni.

\begin{tabular}{|c|c|c|c|c|c|}
\hline \multirow{2}{*}{$\begin{array}{c}\text { Dose } \\
\left.\mathrm{ha}^{-1}\right)\end{array}$} & \multirow{2}{*}{ Concime } & \multicolumn{4}{|c|}{ Livello riempimento tramoggia } \\
\cline { 2 - 6 } & $90 \%$ & $50 \%$ & $10 \%$ & media \\
\hline \multirow{4}{*}{250} & $\mathrm{~A}$ & 5,0 & 19,1 & 12,5 & 12,2 \\
\cline { 2 - 6 } & $\mathrm{B}$ & 10,6 & 10,7 & 29,0 & 16,8 \\
\cline { 2 - 6 } & $\mathrm{C}$ & 8,5 & 6,6 & 10,6 & 8,5 \\
\cline { 2 - 6 } & $\mathrm{D}$ & 0,7 & 3,2 & 5,5 & 3,1 \\
\hline \multirow{4}{*}{500} & $\mathrm{~A}$ & 6,5 & 19,1 & 6,0 & 10,5 \\
\cline { 2 - 6 } & $\mathrm{B}$ & 6,3 & 11,2 & 35,0 & 17,5 \\
\cline { 2 - 6 } & $\mathrm{C}$ & 6,6 & 2,7 & 5,5 & 4,9 \\
\cline { 2 - 6 } & $\mathrm{D}$ & 2,6 & 1,9 & 1,4 & 2,0 \\
\hline \multirow{4}{*}{$\begin{array}{c}\text { Media } \\
\text { dosi }\end{array}$} & $\mathrm{A}$ & 5,8 & 19,1 & 9,3 & 11,4 \\
\cline { 2 - 6 } & $\mathrm{B}$ & 8,4 & 11,0 & 32,0 & 17,1 \\
\cline { 2 - 6 } & $\mathrm{C}$ & 7,5 & 4,6 & 8,0 & 6,7 \\
\cline { 2 - 6 } & $\mathrm{D}$ & 1,7 & 2,6 & 3,4 & 2,6 \\
\hline & Media & 5,9 & 9,3 & 13,2 & 9,5 \\
\hline
\end{tabular}

TABELla 9 - Valori di coefficiente di separazione rilevati per ciascun concime oggetto di prova in relazione alle variabili esaminate.

TABLE 9 - Segregation coefficient obtained as regard to the different examined variables.

\section{CONCLUSIONI}

\section{Conclusions}

La sperimentazione effettuata e la definizione di specifici indicatori e coefficienti ha consentito di valu- tare la qualità di distribuzione di differenti miscele fisiche di concimi e di evidenziare quali delle loro caratteristiche fisiche possono influenzare in maniera negativa l'uniformità di distribuzione. In particolare per quanto riguarda il concime $A$, le differenze di massa volumica tra le frazioni che compongono la miscela hanno determinato sia una certa difficoltà nel mantenere larghezze di lavoro ottimali, sia un elevato valore di indice di differenza tra i diagrammi. D'altra parte, grazie all'uniformità granulometrica delle frazioni, relativamente buona, lo stesso concime è risultato soggetto ad una ridotta separazione nella tramoggia (ridotto valore del coefficiente di separazione). Diversamente il concime $B$, pur risultando caratterizzato da frazioni con masse volumiche che non si differenziano molto tra loro e da un buon indice di differenza tra i diagrammi, è risultato oggetto di una considerevole separazione delle frazioni nella tramoggia (elevato coefficiente di separazione) probabilmente a causa della differente forma dei granuli che compongono le diverse frazioni del concime e in particolare della frazione marrone. Il concime $C$, grazie ad una maggiore uniformità delle classi granulometriche che lo compongono è in grado di fornire buoni risultati, sia in termini di indice di differenza tra i diagrammi, sia di coefficiente di separazione. Infine il concime $D$, essendo composto per il $100 \%$ da granuli con dimensioni $<2,0 \mathrm{~mm}$, non consente di raggiungere elevate larghezze di lavoro, tuttavia proprio grazie alla sua uniformità di classe granulometrica ha fornito sia un sufficiente indice di uguaglianza dei diagrammi sia un ridotto coefficiente di separazione.

In sintesi i risultati ottenuti nella presente ricerca evidenziano, come già rilevato da altri autori $[7,8]$, che i parametri fisici dei concimi ne influenzano la separazione e la qualità della distribuzione. Spesso, però, l'importanza di tali parametri è sottovalutata e la scelta del fertilizzante è effettuata solo sulla base di considerazioni di carattere economico. Si ritiene opportuno, in tal senso, sia approfondire le conoscenze relative all'uniformità di distribuzione e alla separazione delle diverse frazioni dei concimi blended oggi presenti sul mercato, sia definire una serie di parametri standard qualitativi che, una volta indicati in etichetta, consentiranno agli utilizzatori di orientare la scelta del tipo di fertilizzante minerale o di effettuare una corretta taratura degli spandiconcime. Si ritiene che in tal senso un primo contributo possa essere fornito dai parametri qualitativi individuati nel presente lavoro.

\section{BIBLIOGRAFIA}

\section{References}

[1] AA.Vv., Les materiels de Fertilisation et Traitement des cultures - Technologies de l'agriculture, (1997), Collection FORMAGRI.

[2] BALSARI P., TAMAGNONE M., Uno strumento informatico per la taratura degli spandiconcime, Atti $2^{\circ}$ Convegno Nazionale "Informatica e Agricoltura" Firenze (1992),17-18. 
[3] Balsari P., AIroldi G., Spandiconcime: caratteristiche tecniche e modalità d'impiego delle macchine per la distribuzione dei concimi minerali, (1994), Edagricole, Bologna.

[4] Bovolenta S., Pezzi F., Confronto di spandiconcime centrifughi e pneumatici Macchine e Motori Agricoli (1997) 5, 48-53.

[5] CAVAlli R., Analisi della distribuzione di una miscela di fertilizzanti solidi mediante spandiconcime di tipo centrifugo, Macchine e Motori Agricoli (1984) 10, 13-20.

[6] EN 1236, Engrais - Détermination de la masse volumique sans tassement (ISO 3944: 1992 modifiée), Norme européenne (1995).

[7] EN 13739-1, Agricultural machinery - Solid fertilizer broadcasters and full width distributors - Environmental protection - Part 1: Requirements, European Standard (2003).

[8] EN 13739-2, Agricultural machinery - Solid fertilizer broadcasters and full width distributors - Environmental protection - Part 2: Test methods, European Standard (2003).

[9] Fiala M., OBerti R., Prove di un DPAE per il controllo della distribuzione su uno spandiconcime ad azione centrifuga, Atti del VI Convegno Nazionale di Ingegneria Agraria - Ancona (1997), 557-565.

[10] Hofstee J.W., Huisman W., Handling and Spreading of Fertilizers Part 1: Physical Properties of Fertilizer in Relation to Particle Motion, Journal of Agricultural Engineering Research (1990) 47, 213-234.

[11] Hofstee J.W., Handling and Spreading of Fertilizers: Part 2, Physical Properties of Fertilizer, Measuring Methods and Data, Journal of Agricultural Engineering Research (1992) 53, 141-162.

[12] Miserque O., Pirard E., Segregation of the bulk blend fertilizers, Chemometrics and intelligent laboratory systems (2004) 74, 215-224.
[13] Zerulla W., Controlled-release fertilizers and fertilizers with nitrification inhibitor, Bollettino della Società Italiana della Scienza del Suolo (SISS) (2000) 49, 773-786.

\section{Parole chiave:}

Fertilizzanti, Distribuzione, Miscele, Separazione.

\section{SUMMARY}

\section{DISTRIBUTION QUALITY OF BLENDED GRA- NULAR FERTLIZERS}

In order to assess the behaviour of four several blended fertilizers during their distribution a set of trials were performed. Two distribution doses $(250$ and $\left.500 \mathrm{~kg} \mathrm{ha}^{-1}\right)$ and three hopper filling levels $(90 \%$, $50 \%$ and $10 \%$ ) have been compared. The influence of some physical parameters (bulk density and granular distribution) on distribution quality and granular segregation has been analysed by defining two indicators (equality pattern index and segregation coefficient). Results show that fertilizers physical properties have a remarkable influence on segregation and quality distribution. It will be in future necessary to define reference parameters, such as the ones identified in the present study and quality standards for blended fertilizers in order to allow the user to better choice the fertilizer and adjust his spreader.

\section{Key words:}

Fertilizers, Distribution, Blended, Segregation. 